\title{
Correction to: Control in the presence of manipulators: cooperative and competitive cases
}

\section{Zack Fitzsimmons $^{1}$ (D) Edith Hemaspaandra ${ }^{2}$ (D) . Lane A. Hemaspaandra ${ }^{3}$}

Accepted: 30 September 2020 / Published online: 12 October 2020

(c) Springer Science+Business Media, LLC, part of Springer Nature 2020

\section{Correction to: Autonomous Agents and Multi-Agent Systems https://doi.org/10.1007/s10458-020-09475-6}

Unfortunately, a post-galley copyediting error altered the contents of cells in the Condorcet Elections columns of the table in Footnote 7. The corrected table appears below. The original article has been corrected.

\begin{tabular}{|c|c|c|c|c|c|c|}
\hline \multirow[t]{2}{*}{ Control by } & \multicolumn{2}{|l|}{ Plurality } & \multicolumn{2}{|c|}{ Condorcet } & \multicolumn{2}{|l|}{ Approval } \\
\hline & Constr. & Destr. & Constr. & Destr. & Constr. & Destr. \\
\hline Adding candidates & NPC & NPC & $\mathrm{P}$ & $\mathrm{P}$ & $\mathrm{P}$ & $\mathrm{P}$ \\
\hline Deleting candidates & NPC & NPC & $\mathrm{P}$ & $\mathrm{P}$ & $\mathrm{P}$ & $\mathrm{P}$ \\
\hline Adding voters & $\mathrm{P}$ & $\mathrm{P}$ & NPC & $\mathrm{P}$ & NPC & $\mathrm{P}$ \\
\hline Deleting voters & $\mathrm{P}$ & $\mathrm{P}$ & NPC & $\mathrm{P}$ & NPC & $\mathrm{P}$ \\
\hline Partitioning candidates & $\begin{array}{l}\text { TE: NPC } \\
\text { TP: NPC }\end{array}$ & $\begin{array}{l}\text { TE: NPC } \\
\text { TP: NPC }\end{array}$ & $\mathrm{P}$ & $\mathrm{P}$ & $\begin{array}{l}\text { TE: P } \\
\text { TP: P }\end{array}$ & $\begin{array}{l}\text { TE: P } \\
\text { TP: } P\end{array}$ \\
\hline Runoff partitioning candidates & $\begin{array}{l}\text { TE: NPC } \\
\text { TP: NPC }\end{array}$ & $\begin{array}{l}\text { TE: NPC } \\
\text { TP: NPC }\end{array}$ & $\mathrm{P}$ & $\mathrm{P}$ & $\begin{array}{l}\text { TE: } P \\
\text { TP: } P\end{array}$ & $\begin{array}{l}\text { TE: } P \\
\text { TP: } P\end{array}$ \\
\hline Partitioning voters & $\begin{array}{l}\text { TE: P } \\
\text { TP: NPC }\end{array}$ & $\begin{array}{l}\text { TE: P } \\
\text { TP: NPC }\end{array}$ & NPC & $\mathrm{P}$ & $\begin{array}{l}\text { TE: NPC } \\
\text { TP: NPC }\end{array}$ & $\begin{array}{l}\text { TE: } P \\
\text { TP: } P\end{array}$ \\
\hline
\end{tabular}

Publisher's Note Springer Nature remains neutral with regard to jurisdictional claims in published maps and institutional affiliations.

The original article can be found online at https://doi.org/10.1007/s10458-020-09475-6.

Zack Fitzsimmons

zfitzsim@holycross.edu

Edith Hemaspaandra

eh@cs.rit.edu

Lane A. Hemaspaandra

http://www.cs.rochester.edu/u/lane

1 Department of Mathematics and Computer Science, College of the Holy Cross, Worcester, MA 01610, USA

2 Department of Computer Science, Rochester Institute of Technology, Rochester, NY 14623, USA

3 Department of Computer Science, University of Rochester, Rochester, NY 14627, USA 